\title{
ON REGULAR POLYTOPES
}

\author{
Luis J. Boya \\ Departamento de Física Teórica \\ Universidad de Zaragoza, E-50009 Zaragoza, SPAIN \\ luis.boya@gmail.com \\ Cristian Rivera \\ Departamento de Física Teórica \\ Universidad de Zaragoza, E-50009 Zaragoza, SPAIN \\ cristian_elfisico@hotmail.com
}

MSC 05B45, 11R52, 51M20, 52B11, 52B15, 57S25

Keywords: Polytopes, Higher Dimensions, Orthogonal groups

\begin{abstract}
Regular polytopes, the generalization of the five Platonic solids in 3 space dimensions, exist in arbitrary dimension $n \geq-1$; now in dim. 2, 3 and 4 there are extra polytopes, while in general dimensions only the hyper-tetrahedron, the hyper-cube and its dual hyper-octahedron exist. We attribute these peculiarites and exceptions to special properties of the orthogonal groups in these dimensions: the $\mathrm{SO}(2)=\mathrm{U}(1)$ group being (abelian and) divisible, is related to the existence of arbitrarilysided plane regular polygons, and the splitting of the Lie algebra of the $\mathrm{O}(4)$ group will be seen responsible for the Schläfli special polytopes in 4-dim., two of which percolate down to three. In spite of dim. 8 being also special (Cartan's triality), we argue why there are no extra polytopes, while it has other consequences: in particular the existence of the three division algebras over the reals $\mathbb{R}$ : complex $\mathbb{C}$, quaternions $\mathbb{H}$ and octonions $\mathbb{O}$ is seen also as another feature of the special properties of corresponding orthogonal groups, and of the spheres of dimension $0,1,3$ and 7 .
\end{abstract}

\section{Introduction}

Regular Polytopes are the higher dimensional generalization of the (regular) polygons in the plane and the (five) Platonic solids in space. L. Schläfli, studied them around 1850 in higher dimensions, $d>3$ and the complete list of regular polytopes is since then known: the existing regular (in particular, 
convex) polytopes, classified by dimension, are [1]:

2 d) In the plane $\mathbb{R}^{2}$, or inscribed in the circle $\mathbb{S}^{1}$, there are regular polygons $N_{2}$ of any number of sides, $N \geq 3 ; T_{2}=\{3\}$ will be the triangle and $H_{2}=\{4\}$ the square; $\{5\}$ the pentagon, etc.

3d) The five Platonic solids, known since the Greeks: the Tetrahedron $\{3,3\}$, the Cube $\{4,3\}$ and dual Octahedron $(\{3,4\})$, Icosahedron and dual Dodecahedron $\left(Y_{3}\right.$ and $Y_{3}^{*}$; or $\{3,5\}$ and $\{5,3\}$ ); they can also be considered as tessellating by curved surfaces the sphere $\mathbb{S}^{2}$.

4d) Besides the generalization of these five $\left(T_{4}, H_{4}, H_{4}^{*}\right.$, up to $Y_{4}$ and $Y_{4}^{*}$, called "the 120-cell"), there is an extra one, the "24-cell" $\left(\equiv\left(H_{4}^{*} H\right)\right)=\{3,4,3\}$.

n-d): Only the Tetrahedron, the Cube and the dual Octahedron generalize, so $T_{n}, H_{n}$ and $H_{n}^{*}$ exist for any $2 \leq n<\infty$ (although $H_{2} \approx H_{2}^{*}$ ), and they are the ones unique for $d>4$.

In the spirit of J. Stillwell's [2] study of exceptional objects in mathematics, one should ask why do these regular polytopes exist, in particular why the exceptions in dimensions 2, 3 and 4. To understand this is the purpose of this paper, written mainly for physicists.

We shall argue that the special properties of the isometry (in particular rotation) groups in Euclidean geometry in dimensions 2 and 4 is the reason for the extra polytopes: in dim. two the rotation group $\mathrm{SO}(2)$ is (abelian and) divisible, (defined below), and in dimension four the covering group $\operatorname{Spin}(4)=\mathrm{SO}(4)^{\sim}$ is not simple, but only semisimple. Also, in dimension eight the $\operatorname{Spin}(8)$ group enjoys also a special property, namely triality, and we shall argue why this does not produce other regular polytopes, but shows itself in other features.

The plan of this work is the following: In Sect. II we set the stage and recall the generic construction of $T_{n}, H_{n}$ and $H_{n}^{*}$ in arbitrary dimension; we shall consider the $n$-dim polytopes as living in $\mathbb{R}^{n}$ and tessellating the $(n-1)$-dim sphere, as $\mathbb{S}^{n-1} \subset \mathbb{R}^{n}$. We also consider the discrete isometry and rotation invariance groups of these polytopes, and the binary extensions of the later groups $\mathrm{SO}(n)$ to the Spin groups, $\operatorname{Spin}(n)$.

In Sect. III we shall consider dimension two, with the existence of the field of complex numbers $\mathbb{C}$ and the regular polygons of arbitrary number of sides $N \geq 3$ a consequence of the $\mathrm{SO}(2)=\mathrm{U}(1) \approx \mathbb{S}^{1}$ group being (abelian and) divisible. 
In Sect. IV we shall work out the three exceptional Schläfli polytopes $\left(Y_{4}\right.$, its dual $Y_{4}^{*}$ and self-dual $\left.\left(H_{4}^{*} H\right)\right)$ in $\operatorname{dim} 4$ as well as the quaternion division algebra $\mathbb{H}$ as a consequence of the split character of the Lie algebra of the orthogonal group $\mathrm{O}(4)$; by an isotropy (stabilizer) argument we shall also encounter the two "old" extra 3 -dim. polyhedra $Y_{3}\{3,5\}$ and $Y_{3}^{*}\{5,3\}$.

In Sect. $\mathrm{V}$ we shall (briefly) consider dimension 8, arguing for the triality of the $\operatorname{Spin}(8)$ group, with the octonion division algebra $\mathbb{O}$, and other consequences like parallelizability of the $\mathbb{S}^{7}$ sphere, and we shall also argue why this does not generate new polytopes.

Some final comments and open questions are left for our final Sect. VI.

There is plenty of literature on the subject. In particular, we may refer here to some pertinent work by Coxeter $([1,3])$, [4] and the recent book [5]). For a view of quaternions and octonions close to ours, but not identical, see [6]. The most up-to-date reference on regular polytopes is [7].

\section{General Polytopes}

We take as known the precise definition of regular polytopes [1] we emphasize here already that we consider only regular polytopes, necessarily convex, and perhaps $n$-dimensional. They can be seen existing as individual solids in $\mathbb{R}^{n}$, but also more appropriately as tessellating a circumscribed sphere $\mathbb{S}^{n-1}$ (there are, as we shall see, other spheres of interest). The Euler characteristic $\chi$ of theses spheres is computed (since Euler and Poincaré) as the alternative sum of the number of faces: if the polytope has $V$ vertices, $A$ edges, $F$ surfaces, $C$ cells and in general (last) objects (boundary polytopes) $S$, the vertices scatter through the outer sphere, the edges become arcs of geodesics, the surfaces are curved, etc. For this outer sphere, containing the vertices, we have

$$
\begin{array}{ll}
\chi\left(\mathbb{S}^{2 n}\right)=2=V-A+F-C \ldots+S & \left(\text { e.g. } 4-6+4 \text { for the } T_{3}\right) \\
\chi\left(\mathbb{S}^{2 n+1}\right)=0=V-A+F-C \ldots-S & \left(\text { e.g. } 5-10+10-5 \text { for } T_{4}\right)
\end{array}
$$

If one includes the improper sets, say the empty set and the whole polytope, then the Euler characteristic is always 0 regardless of whether $n$ is odd or even.

Starting in dim one, the segment $I:-$, has the 0-sphere $\circ \circ$ as boundary, $\partial I=S^{0}=\mathbb{S}^{0}$ : just two vertices can be considered as the only "regular" polytope in dimension one. One can consider also the 0 -sphere as 
the set of norm-one real numbers in $\mathbb{R}$. Let $l$ be the (arbitrary) length of $I$, between the two circles.

To construct the generic regular polytopes $T_{n}, H_{n}$ and $H_{n}^{*}$ : we first draw (for $n=2$ ) in the plane $\approx \mathbb{R}^{2}$ the perpendicular bisector to the segment $I$ until its distance from the top to the two vertices equals the length $l$ : putting in the edges, we have then the ordinary (plane) regular triangle $T_{2}=\{3\}$. Also a parallel line to $I$ at distance $l$ in the same (bi-)plane closes down to the square $H_{2}=\{4\}$; and if in the triangle case, we extend symmetrically the mid-perpendicular below $I$, we construct the rhomb as the "2-dim" dual of the square, $H_{2}^{*}$, which in fact is isomorphic with the square itself (only in this dimension two). As $\chi\left(S^{1}\right)=0$, we have $3-3$ and $4-4$ for the vertices $V$ and edges $A$, while the rotation symmetry groups are obviously $\mathrm{Z}_{3}$ and $\mathrm{Z}_{4}$ respectively. Denoting by $\{p\}$ the general regular polygon of $p$ edges (Schläfli and Coxeter notations), whose existence we shall justify, we have $p-p$ as $V-A$ for regular polygons, for any $p \geq 3$. One sees also that all polygons are trivially "self-dual": changing vertices by edges we get the same figure; also the (inner) circle $S^{1}$, tangent to the mid-edges is like the outer one, rotated $2 \pi / 2 N=\pi / N$ from the plane polygon $N_{2}$.

One is easily convinced that this triple procedure generalizes to any higher dimension $n>2$, generating hyper-tetrahedron $T_{n}=\{3,3, \ldots 3\}$, hyper-cube $H_{n}=\{4,3,3,3 \ldots 3\}$ and dual hyper-octahedron $H_{n}^{*}=\{3,3,3 \ldots 3,4\}$; for the tetrahedron $T_{3}$, for example, we draw in space the normal through the centre of a regular plane triangle $3_{2}=T_{2}=\{3\}$ and link the top vertex with the existing 3 plane vertices to form the usual tetrahedron $T_{3}$; or draw a plane parallel to the square and above it to form the cube $H_{3}$; or again prolonging the normal below the plane of the triangle we get the octahedron $H_{3}^{*}$, now $\neq H_{3}$. Recall trivially the number of faces in 3 dimensions:

$$
\begin{aligned}
& \text { For } T_{3}: \quad V-A+F=4-6+4 ; \quad \text { For } H_{3}: \quad V-A+F=8-12+6 ; \\
& \text { For } H_{3}^{*}: \quad V-A+F=6-12+8 ;
\end{aligned}
$$

Again, the Schläfli-Coxeter notation $\{p, q\}$ means (in 3-dim): surfaces of $p$ edges, and $q$ edges per vertex: hence $\{3,3\},\{4,3\}$ and $\{3,4\}$ are $T_{3}, H_{3}$ and $H_{3}^{*}$ respectively. Here $T_{3}$ is self-dual, whereas $H_{3}$ and $H_{3}^{*}$ are duals of each other. In these $3 d$ case we have three 2 -spheres, the "middle one" $S_{m}^{2}$ being tangent to the mid-edges.

The generalization to higher dimension is obvious in these three cases. One just adds either a normal line or a parallel (hyper-)plane to get $T_{n+1}$, $H_{n+1}$ and $H_{n+1}^{*}$ from the previous, $n$-dim case. For the $T_{n+1}$, we have e.g. $n+2$ vertices connected with each other by $\left(\begin{array}{c}n+2 \\ 2\end{array}\right)$ edges, ..., up to 
$\left(\begin{array}{l}n+2 \\ n+1\end{array}\right)=n+2$ cells $S$. Besides the outer and the inner spheres, tangent respectively to vertices and cells, we have several intermediate ones (see below). For faces for $H_{n}, H_{n}^{*}$ see e.g. [1].

We shall not prove (Schläfli) that there are NO other regular polytopes $\Pi$ in arbitrary dimension, but just quote the usual argument for three: for dim. 3 the five Platonic solids exhaust all the possible ones (for a short argument in dim. 4 see [18], pag. 396): if there are $p$ edges per surface $(F$ of them) and $q$ edges from each vertex $(V)$, from Euler's $\chi=V-A+F=2$ we get $\frac{1}{p}+\frac{1}{q}=\frac{1}{2}+\frac{1}{A}$, or $\frac{1}{p}+\frac{1}{q}>\frac{1}{2}$, with the three possible solutions $(p, q)$ $=\{3,3\},\{3,4\},\{3,5\}$, plus the two duals, that is $T_{3}, H_{3}, H_{3}^{*}$ and the two extra $Y_{3}, Y_{3}^{*}$. If $A=\infty, \frac{1}{p}+\frac{1}{q}=\frac{1}{2}$ would correspond to tessellations of the (non-compact) plane $\mathbb{R}^{2}$ (see Sect. VI), with solutions (Kepler): triangles $\{3,6\}$, dual hexagons $\{6,3\}$ and self-dual squares $\{4,4\}$.

Now for the symmetry. In Euclidean space $\mathbb{R}^{n}$ we can consider the Euclidean group $\mathrm{E}(n)$, with translations, rotations and reflections, $\mathrm{E}(n) \approx$ $T_{n} \rtimes \mathrm{O}(n)(A \rtimes B$ is our conventional notation for the semidirect product, with $A$ normal, acted upon by $B)$. As polytopes have a centre, there are no translations in their isometry groups. Let us call $\mathrm{O}(n)$ the orthogonal group, and $S O(n)$ the rotation subgroup: the later is a connected, compact $\frac{n(n-1)}{2}$ dim. Lie group, $n \geq 2$. Recall, though, this rotation group is not simply connected, and (for $n \geq 3$ ) has a double universal cover, called Spin(n). The full structure of the orthogonal group is therefore given by

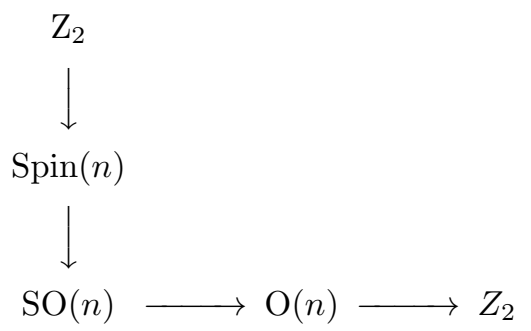

It is easy to see the universal cover in the $3 \mathrm{~d}$ case (the argument is standard in physics); namely, the ordinary rotation group $\mathrm{SO}(3)$ contains rotations $<2 \pi$ around any axis: one parameterizes direction axis by points in $S^{2}$, and rotation angle with the radius: hence $\mathrm{SO}(3) \approx B_{3}$, the solid 3-ball of radius $\pi$, except that antipodal points in the boundary sphere are rotations differing in $2 \pi$ along the same direction, so they coincide. The topology of the rotation manifold is therefore that of $\mathbb{R} P^{3}$, as the $n$-ball is like a half $n$-sphere, and with the antipodal identification becomes the projective space. Hence, $\pi_{1}(\mathrm{SO}(3))=\pi_{1}\left(\mathbb{R} P^{3}\right)=\mathrm{Z}_{2}$, and one shows the result holds in general, $n \geq 3$.

What about the (discrete) isometry and rotation groups for our polytopes? It is also easy to compute them: we attack directly the $n$-dim. case: 
$T_{n}$, hypertetrahedron in $n$ dimensions: $V=n+1$, and, as any vertex links with any other, edges $A=\left(\begin{array}{c}n+1 \\ 2\end{array}\right)$; surfaces $F=\left(\begin{array}{c}n+1 \\ 3\end{array}\right) \ldots ; S=\left(\begin{array}{c}n+1 \\ n\end{array}\right)$. The Rotation group is Alt $_{n+1}$, the Isometry group $\operatorname{Sym}_{n+1}$ : an arbitrary permutation of the vertices is the most general isometry, as any vertex connects with any other by some edge; then rotations alone generate the even permutations, the alternative $\mathrm{Alt}_{n+1}$. We can write this result in the bundle form for the rotation part as tessellating the outer sphere, itself an homogeneous space under the total rotation group $\mathrm{SO}(n)$ with the result

$$
\begin{aligned}
& \operatorname{Alt}_{n} \longrightarrow \operatorname{Alt}_{n+1} \longrightarrow T_{n} \\
& \cap \\
& \mathrm{SO}(n-1) \longrightarrow \mathrm{SO}(n) \longrightarrow S^{n-1}
\end{aligned}
$$

(The inclusions can be seen as injective arrows). Notice, for example, that $\mathrm{Alt}_{3}=\mathrm{Z}_{3}$ abelian maps injectively into $\mathrm{SO}(2)$, abelian as well. Also, these hypertetrahedra are self-dual in the change $p$-faces by $(n-p)$ faces (see above). In the short notation, $T_{n}$ is $\left\{p,\left(\begin{array}{c}p \\ 2\end{array}\right), \ldots,\left(\begin{array}{c}p \\ p-1\end{array}\right)\right\}$. Notice (4) applies to vertices or the last faces $S$, as $T_{n}$ is self -dual under the interchange of $p$-faces with $(n-p)$ faces described above. But the rotation symmetry group can act also in the intermediate faces, e.g. in the edges: if it just permutes or flips the edges, the operation is like a $\pi$ rotation, so the upper line above becomes $\mathrm{Z}_{2} \longrightarrow$ Alt $_{n} \longrightarrow$ (edges), and one sees the known result [1] that the order of the finite rotation group in $3 \mathrm{~d}$ is twice the number of edges, $\left|\operatorname{Rot} \prod\right|=2 A$.

For the hypercube $H_{n}$, and dual $H_{n}^{*}$ it is easy to see that the full isometry group permutes the $n$ axes of an orthonormal frame $\left\{e_{1}, \ldots, e_{n}\right\}$, and besides one can reflect each of them on the origin, $e_{i} \longrightarrow-e_{i}$ : for the square $\square$, the rotation group is $Z_{4}$ whereas the isometry group (adding reflections) is the dihedral $D_{4}=Z_{4} \rtimes Z_{2}$. One sees this construction permutes the $n$ basis of a frame, plus the inversions of the same, so the isometry group is

$$
\operatorname{Isom}\left(H_{n}\right)=\operatorname{Isom}\left(H_{n}^{*}\right)=\left(\mathrm{Z}_{2}\right)^{n} \rtimes \operatorname{Sym}_{n} \quad\left(\text { order } 2^{n} \cdot n !\right)
$$

For example, for dim 2, 3,4 the order of the full group is 8,48 and 384 , and the order of the rotation part is half. E.g. the real structure of the rotation groups is $Z_{4}$, Sym $_{4}$ and $\left(\mathrm{Z}_{2}\right)^{3} \rtimes \mathrm{Sym}_{4}$, orders 4, 24 and 192 ( for example, $\operatorname{Rot}\left(H_{3}\right)=\mathrm{Sym}_{4}$, permuting the four diagonals).

The binary extensions of the rotation groups were already used back in the 19-th century [3]. The $\operatorname{Spin}(n)$ group is the universal cover of $\mathrm{SO}(n)$ for $n \geq 3$. The later notation $2 \cdot G$ is used by J. H. Conway (thus the $\mathrm{SO}(n) \cdot 2$ is the one for the full orthogonal group $\mathrm{O}(n)$ ). So we can now write the binary extension in the previous form, e.g. for the $n$-Cube $H_{n}$ :

$$
\begin{aligned}
& 2 \cdot G_{0} \longrightarrow \mathrm{G}_{0}:=\mathrm{Z}_{2}^{n-1} \rtimes \mathrm{Sym}_{n} \longrightarrow H_{n} \\
& \cap \\
& \mathrm{Spin}(n) \longrightarrow \mathrm{SO}(n) \longrightarrow S^{n-1}
\end{aligned}
$$

The standard name for these $2 \cdot$ extensions is "binary": Thus $2 \cdot$ Alt $_{n+1}$ is called (for $n \geq 3$ ) the "binary tetrahedral" group, etc. 
The faces for the hyper-cube are easily calculated, also; for example:

For $H_{4}$, they are $(8+8,16 \times 4 / 2,24,2+6=16-32+24-8)$

For $H_{5}^{*}$, they are $(10,40,80,80,32)$

\section{Dimension two: complex numbers and polygons of $n$ sides}

The isometry group of the plane $\mathbb{R}^{2}$ is the Euclidean group $\mathrm{E}_{2}=\mathbb{R}^{2} \rtimes \mathrm{O}(2)$. For a polygon, the centre is fixed, so the symmetry group is inside the orthogonal group $\mathrm{O}(2)$, and the connected (rotation) part is in $\mathrm{SO}(2)=\mathrm{U}(1)$. This group is abelian (the general rotation group $\mathrm{SO}(n), n>2$ is not, of course), and divisible in the category of abelian groups, that is, under any discrete cyclic subgroup $Z_{n}$ the quotient is still $\mathrm{U}(1)$ :

$$
\mathrm{U}(1) / \mathrm{Z}_{n} \approx \mathrm{U}(1)
$$

We recall: In the category $\mathcal{A} b$ of abelian groups [19, the projective objects are the groups $\mathrm{Z}^{n}$, with $n \mathrm{Z} \approx \mathrm{Z}$, whereas the divisible ones (= injectives) verify $\mathrm{U} / \mathrm{Z}_{n} \approx \mathrm{U}$ : this is also the reason why the "double cover" of the plane rotation group, $\mathrm{SO}(2)$ is isomorphic with the original, $\mathrm{SO}(2)$ group; recall, in this case, the universal cover has "infinite" sheets, as $\pi_{1}\left(S^{1}\right)=Z$, and the covering sequence is $\mathrm{Z} \longrightarrow \mathbb{R} \longrightarrow S^{1}$. As the crucial fact for our next construction (of the complex numbers $\mathbb{C}$ as well as the regular polygons) is that the 1 -sphere $S^{1}$ can be endowed with a group structure, we add the following simple argument: when $\mathrm{O}(n)$ operates in $S^{n-1}$, the action is still transitive, with stabilizer $\mathrm{O}(n-1)$; hence, for $\mathrm{O}(2)$ acting on $S^{1}$, the isotropy group is $\mathrm{O}(1)=\mathrm{Z}_{2}$; going to rotations, $\mathrm{SO}(2)$ is still transitive on the circle, with stabilizer $\mathrm{SO}(1)=1$. Hence $S^{1} \approx \mathrm{SO}(2)$, acquires an (abelian and divisible, as $\mathrm{SO}(2) \approx \mathrm{U}(1))$ group structure!

This produces several consequences: first, one can see the complex numbers as an emerging structure in $\mathbb{R}^{2}$ in this way: write $\mathbb{R}^{2} \backslash\{0\}$ as $S^{1} \times R^{+}$, as the polar coordinates in the plane, where $S^{1} \approx \mathrm{U}(1)=\mathrm{SO}(2)$; notice the two groups $\mathrm{U}(1)$ and $R^{+}$are abelian and commute, and establish then the multiplication law $(\approx$ for complex numbers $\neq 0):(\phi, r) \cdot\left(\phi^{\prime}, \mathrm{r}^{\prime}\right):=\left(\phi+\phi^{\prime} \bmod 2 \pi, \mathrm{rr}^{\prime}\right)$. Add now the origin 0 with $0 \cdot(\phi, r):=0$ : we have the complex numbers $\mathbb{C}$ with the usual invertible multiplication: $(\phi, r)^{-1}=\left(-\phi, \frac{1}{r}\right)$ (the addition is the normal one for "vectors" in $\mathbb{R}^{n}$ for $\left.n=2\right)$. We leave to the reader to check the distributive law: $\mathrm{z}\left(\mathrm{z}^{\prime}+\mathrm{z}^{\prime \prime}\right)=$ $\mathrm{zz}^{\prime}+\mathrm{zz}^{\prime \prime}$. The moral:

Both the existence of the field of complex numbers $\mathbb{C}$ and the existence in the plane of regular polygons $N_{2}$ of any arbitrary number of sides $N$ come from the following fact: that the rotation group $\mathrm{SO}(2)$ is abelian and divisible... and that the 1-sphere $S^{1}$ has a group structure: The abelian character of $\mathrm{SO}(2)$ guarantees the existence of the field of complex numbers (recall: commutativity is a property of the modern definition of a field). But also implies the existence of the regular polygons: the previous equation (7) just means one can inscribe a regular polygon 
of $n$ sides in the circle. This result is so trivial that usually one never bothers asks why regular polygons do exist with any number of sides.

For completeness, we express the rotation subgroup of the symmetry group for an arbitrary (plane) polygon $N_{p}$, as embedded in the rotation \& orthogonal groups as

$$
\begin{aligned}
& \mathrm{Z}_{p} \approx N_{p} \quad \mathrm{Z}_{2} \longrightarrow \mathrm{D}_{p} \longrightarrow \mathrm{N}_{p} \\
& \cap \cap \text { and } \| \quad \cap \quad \cap \\
& \mathrm{SO}(2) \approx S^{1} \quad Z_{2} \longrightarrow \mathrm{O}(2) \longrightarrow S^{1}
\end{aligned}
$$

where $\mathrm{D}_{n}=\mathrm{Z}_{n} \rtimes \mathrm{Z}_{2}$ is the dihedral group.

Although the binary groups exist, they are not the universal cover, because $\pi_{1}(\mathrm{SO}(2))=\pi_{1}\left(S^{1}\right)=\mathrm{Z}$, and we shall not consider them.

\section{Dimension four: the Lie algebra of $O(4)$ group splits}

For any Lie group $\mathrm{G}$ we know its Lie algebra, written $L(G)$, which is the algebraic structure of vectors in the tangent space to the identity of the group (a Lie group is by definition also a manifold) propagated along the whole group by the very same group action: so the algebra is generated by (e.g.) the left-invariant vector fields at the identity, and has the dimension of the group (as a real vector space).

The complex simple Lie algebras were classified by Cartan back in 1894 . He also wrote some identities among small orders, for example $A_{1}=B_{1}=C_{1}$, that is, in the compact group form $\mathrm{SU}(2)=\operatorname{Spin}(3)=\mathrm{SpU}(1)$; the last of the four Cartan identities says that $D_{2}=B_{1}+B_{1}$, or $\operatorname{Lie}(\mathrm{O}(4))=\operatorname{Lie}(\mathrm{O}(3))+\operatorname{Lie}(\mathrm{O}(3))$. As this is the property we need in our construction, we supply two different proofs of the split character of the $L[\mathrm{O}(4)]$ : an algebraic proof, and a topological one; (Cartan's simpler proof was from the Diagram $D_{2} \approx \circ \quad \circ$ (for Dynkin diagrams see e.g. [10]).

1) Algebraic proof: The rotation group is unimodular, $\mathrm{SO}(n) \subset \mathrm{SL}_{n}(R)$; hence its representations in $p$-forms and in $(n-p)$-forms are equivalent. On the other hand, the Lie algebra of $\mathrm{O}(n)$ is realized by all antisymmetric matrices, as ${ }^{t} o o=$ $1 \Longrightarrow L=-{ }^{t} L$, where $o(t):=\exp (t L)$. Therefore, for $\operatorname{dim} 4$ and only for that, the Lie algebra of the group splits: $\mathrm{Lie}[\mathrm{O}(4)]=$ self-dual part plus anti-self-dual, or 6 $=3+3$, or

$$
\mathrm{Lie}[\mathrm{O}(4)] \approx \mathrm{Lie}[\mathrm{O}(3)] \oplus \operatorname{Lie}[\mathrm{O}(3)]
$$

At the level of groups, the equations are, for example

$$
\operatorname{Spin}(4)=\operatorname{Spin}(3) \times \operatorname{Spin}(3)
$$

2) The topological second proof is given in the bundle language: write the principal bundle $(G, B, M)$ as

$$
\mathrm{SO}(3) \longrightarrow \mathrm{SO}(4) \longrightarrow S^{3}
$$


i.e., the action of $\mathrm{SO}(n)$ in the $(n-1)$ sphere for $n=4$ : the case for $n=3$ is wellknown. Now the G-bundles $B$ over spheres $S$ are classified [11] by some homotopy group: if we write

$$
G \longrightarrow B \longrightarrow S^{n}
$$

then the set of $G$-bundles over $S^{n}$ is $H^{1}\left(S^{n}, G\right)$ in Čech cohomology, and is given by

$$
H^{1}\left(S^{n}, G\right)=\pi_{n-1}(G)
$$

(essentially because one trivializes the sphere by two charts overlapping in the equator). Now a fundamental result proved by Cartan [12] is that

$$
\pi_{2}(G)=0
$$

for any (finite dimensional) Lie group G (intuitively, the topology of Lie groups is given by that of products of odd dimensional spheres [13]). Hence, in our case $\mathrm{SO}(4) \approx \mathrm{SO}(3) \times S^{3}$ (not as product of groups: $\mathrm{SO}(3)$ is not normal in $\mathrm{SO}(4)$ !) which clearly amounts to our result

$$
\mathrm{Lie}[\mathrm{O}(4)]=\mathrm{Lie}[\mathrm{O}(3)]^{2}
$$

As expected from the $2 d$ case, both the existence of the quaternion division algebra $\mathbb{H}$ (not a field, but a skew-field: quaternion product (Hamilton, 1842) is not commutative!) and the extra Schläfli polytopes (in 4-dim.) are a consequence of this: Let us first show the quaternion division algebra; it should be already clear to the reader that

$$
S^{3} \approx \mathrm{SU}(2)=\operatorname{Spin}(3):=\mathrm{SO}(3)^{\sim}=2 \cdot \mathrm{SO}(3)=\mathrm{SpU}(1)
$$

(One writes $\mathrm{SU}(2)$ being unitary and unimodular, with centre $Z_{2}$ ). So we can now sort of repeat the construction of the complex case: we proved the crucial fact, that the 3 -sphere $S^{3}$ admits also (like $S^{1}$ ) a (non-commutative!) group structure; the simplest proof: $\mathrm{SU}(2)$ acts transitive in the sphere $S^{3} \subset \mathbb{R}^{4}=\mathbb{C}^{2}$ with stabilizer $\mathrm{SU}(1)=\mathrm{I}$; next, in $\mathbb{R}^{4} \backslash\{0\}$ we form the direct product of groups, as before

$$
\mathbb{R}^{4} \backslash\{0\} \approx S^{3} \times \mathbb{R}^{+}
$$

with again the composition law $(\boldsymbol{\theta}, r)\left(\boldsymbol{\theta}^{\prime}, r^{\prime}\right)=\left(\boldsymbol{\theta} \cdot \boldsymbol{\theta}^{\prime}, r r^{\prime}\right)$, and adding again the 0 as above (in the complex case) we have formed the division algebra of Hamilton quaternions! (In particular, the inverse of $(\boldsymbol{\theta}, r) \neq 0$ is $\left(\boldsymbol{\theta}^{-1}, \frac{1}{r}\right)$. Here $\boldsymbol{\theta}$ is a label of the 3-parameter element of $\mathrm{SU}(2)$. Hence, $\boldsymbol{\theta} \cdot \boldsymbol{\theta}^{\prime}$ is explicit in the multiplication in $\mathrm{SU}(2)$. Of course, $S^{3}$ becomes then the group of unit quaternions, and the "equator" $S^{2}$ (no longer a group!) can be identified with the set of unit imaginary quaternions also. So we see, as announced, that the quaternions owe its existence to the $\operatorname{Spin}(4)$ group being only semisiple, not simple, what makes also the 3 -sphere a Lie group: Another expression of the same thing, would be that it is the product (group) structure in the odd spheres $S^{1}$ and $S^{3}$ which is ultimately responsible for the field of the complex numbers $\left(S^{1}\right)$ and the skew-field of the quaternions $\left(S^{3}\right)$ : later we shall show how the "sphere" $S^{0}$ with just two points and the seven-sphere $S^{7}$ are also associated with the real field $\mathbb{R}$ and the octonion division algebra $\mathbb{O}$, 
respectively.

Now for the extra polytopes: In the hypertetrahedron $T_{4}=\{3,3,3\}$, which exists in arbitrary dimension, we have the rotation diagram

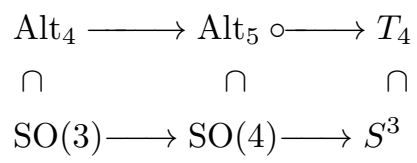

As $\mathrm{SO}(4)$ splits, the image of $\mathrm{Alt}_{5}$, which is a simple group, must go in one of the factors, so it must be $\mathrm{SO}(3)$, which is (sub)group: we have then a regular polytope in 3-dim with rotation symmetry Alt $_{5}$, so we have a new polytope in 3 dimensions! (notice $Z_{4}$ is not contained in $\mathrm{Alt}_{5}$ ):

$$
\begin{aligned}
& \mathrm{Z}_{i} \longrightarrow \mathrm{Alt}_{5} \longrightarrow Y_{3} \\
& \cap \cap \cap \cap \\
& \mathrm{SO}(2) \longrightarrow \mathrm{SO}(3) \longrightarrow S^{2}
\end{aligned}
$$

$\left(\mathrm{Z}_{i}, i=2,3\right.$ or 5$)$ That is to say, the action $\mathrm{SO}(3)$ to $S^{2}$ has the abelian $\mathrm{SO}(2)$ as stabilizer, hence one should ask for an abelian subgroup of Alt ${ }_{5}$, and the two more useful ones are $\mathrm{Z}_{3}$ and $\mathrm{Z}_{5}$ : this is the rotation symmetry of either triangular or pentagonal faces, so we have constructed both the icosahedron $Y_{3}$ and the dual dodecahedron $Y_{3}^{*}$ ! Notice that we have to use the fact that polygons of arbitrary (five, three ...) number of edges exist in dimension two! Also, the subgroup $Z_{2}$ corresponds to the involutions on the edges, see just below.

We generate therefore the icosahedron $Y_{3}=\{3,5\}$, with $V-A+F=12-30+20$, and the dual dodecahedron $Y_{3}^{*}=\{5,3\}$ with $20-30+12$. Notice, as $V+F=32$ in both cases, the number of edges is fixed to 30: the two exotic Platonic solids are being explained (we shall see them again as coming from four dimensions!). Notice even the subgroup $Z_{2}$ in $A_{1 t}$ : it occurs when the Alt 5 group acts on the $30\left(=\frac{60}{2}\right)$ edges of either $Y_{3}$ or $Y_{3}^{*}$.

But there is more: the extra polytopes in dimension four!

As $A l t_{5}$ lies in $\mathrm{SO}(3)$, it can be "lifted" to $2 \cdot A l t_{5}$, order 120 , by going to the covering $S_{3} \approx \mathrm{SU}(2)=\operatorname{Spin}(3)$. Hence we have a 120 -vertex tessellation of $S^{3}$, one of the Schläfli new polytopes! The situation is this:

$$
\begin{aligned}
& \mathrm{Alt}_{5} \longrightarrow G_{120 \times 60} \longrightarrow Y_{120} \\
& \cap \\
& \mathrm{SO}(3) \longrightarrow \mathrm{SO}(4) \longrightarrow S^{3}
\end{aligned}
$$

But $\mathrm{Alt}_{4}$ acts on the $T_{3}$ polytope in $3 \mathrm{~d}$, so it is also a subgroup of $\mathrm{SO}(3)$. Then

$$
\begin{aligned}
& \mathrm{Alt}_{4} \longrightarrow G_{600 \times 24} \longrightarrow Y_{600} \\
& \cap \\
& \cap
\end{aligned}
$$


The construction is similar to the icosahedron/dodecahedron in $3 d$ :

To complete the enumeration of the faces, we argue in the following way: from the $Y_{3}$, the $3 d$ icosahedron, $12-30+20$, we have $20 \times \frac{3}{2}=30$ edges, so now the number of edges per vertex is one more, and we have $600 \times \frac{4}{2}=1200$ edges, so, as $\chi=0$, the two new potytopes have:

$$
Y_{4}: 120,720,1200,600, \quad \text { and } Y_{4}^{*} \text { the dual, 600,1200,720,120. }
$$

In other words, we "understand" both the 120-cell and the 600 cell. We can even retrieve the $3 d$ polyhedra $Y_{3}$ and $Y_{3}^{*}$ by fixing a vertex, getting $12-30+20$ for $Y_{3}$ and $20-30+12$ for the dual $Y_{3}^{*}$, as we know already. The real novelty is the appearance of the pentagons, both in $3 d$ and in $4 d$. In Schläfli notation, we have

$\{3,3,3\}$ for $T_{4},\{4,3,3\}$ and dual $\{3,3,4\}$ for $H_{4}$ and $H_{4}^{*} \cdot ;\{3,3,5\}$ for the 600 cell $Y_{4}$, and dual $\{5,3,3\}$ for the 120 -cell, $Y_{4}^{*}$.

So we "understand" five of the six regular polytopes in 4 dimensions. What about the remaining one, called "the 24-cell"? Here, unfortunately, we do not have such a "pentagonal" argument, but a simpler one, characteristic also of dimension four, which does NOT descend to three. This is our construction:

Compare the hypercube $H_{4}(+16-32+24-8)$ with the dual hyper-octahedron $H_{4}^{*}(8-24+32-16)$ : the relation, in the square, between centre and half-side vs. centre and corner is $\sqrt{2}$. In the ordinary cube, is $\sqrt{3}$ and in the $H_{4}$ is 2 $=\sqrt{1+1+1+1}$ : Hence, the vertices of the hyperoctahedron are at the same distance (2) from the vertices of the hypercube themselves!. Hence, a unique new polytope is possible in four dimensions, made out of the two: each vertex combines with four others form the $h$-cube, and with another 4 from the $h$-octahedron, to produce $8+16$ vertices, and $24 \cdot \frac{8}{2}$ edges, etc.:

The 24-cell : $\quad(24,96,96,24), \quad$ which is self-dual

In the Coxeter-Schläfli notation, the 24 -cell is clearly $\{3,4,3\}$. It only remains to compute the isometry groups: one sees "easily" that one should include three new rotations, for the triangular surfaces of the octahedron:

$\mid \operatorname{Isom}(24-$ cell $)|=3 \cdot| \operatorname{Isom}\left(H_{4}\right) \mid=1152$

which coincides, as it should, with the Weyl Group of the second exceptional Lie Group $F_{4}$ (private communication from Joseph C. Varily).

\section{Dimension 8: triality}

Dimension 8 is also special (if only because the existence of octonions!). The characteristic property is triality, already discovered by É. Cartan in 1925; namely, the centre of the $\mathrm{SO}(8)$ group is $\mathrm{Z}_{2}$, as in any even dimensional rotation group; so the $\operatorname{Spin}(8)$ group has centre of order four, again as in any $\operatorname{Spin}(2 \mathrm{n})$ group: but, from Bott periodicity [20, we know: for $\operatorname{Spin}(4 n+2)$ the centre is $Z_{4}$ (e.g. $\operatorname{Spin}(6)$ $=\mathrm{SU}(4))$, and for $\mathrm{Spin}(4 \mathrm{n})$ the centre is $\mathrm{V}=\left(\mathrm{Z}_{2}\right)^{2}$. For $\operatorname{dim} 8 n+4$ the two spinor representations are quaternionic, that is, complex, and conjugate equivalent, but 
not possible in real form. But in $\operatorname{dim} 8 n$ they are both real, and for $\operatorname{dim} 8$ (and only for that!) the $\operatorname{dim}$ is also $8=2^{\frac{8}{2}-1}$ : the three irreps namely vectorial $\square$, and the two chiral ones, $\Delta_{L}$ and $\Delta_{R}$ are real and of dimension 8: they can be interchanged under a $S_{3}$ symmetry: that is the original Cartan's triality (on the other hand, a very obvious symmetry from the Dynkin diagram). Notice the quoted centre $V=\left(Z_{2}\right)^{2}$ also plays a role: these three irreducible representatations are not faithful, with a $Z_{2}$ kernel, different in each case: the three $Z_{2}$ subgroups of the center group, $\mathrm{V}=\left(\mathrm{Z}_{2}\right)^{2}$ (in any group $\mathrm{G}$, if there are more than one central involution, no irrep can be faithful, as Schur's lemma forces these involutions to be represented by \pm 1$)$. It is remarkable that $\operatorname{Aut}($ Center $=\mathrm{V})=S_{3}=\operatorname{Out}(\operatorname{Spin}(8))$, but the two groups seem to have nothing to do with each other! This, in spite of the morphism between outomorphisms of the group and automorphisms of the center: Out $(\mathrm{G}) \longrightarrow \operatorname{Aut}\left(\mathrm{Z}_{G}\right)$ for any group $\mathrm{G}$.

There is a slight generalization of triality, discussed by J. F. Adams [14; namely in any algebra, there is a product in the underlying vector space: $V \times V \longrightarrow V$. In the form $V \times V \times V \longrightarrow 1$, it is Adams's triality: in particular it holds for the four division algebras $\mathbb{R}, \mathbb{C}, \mathbb{H}$ and $\mathbb{O}$. In our case, one shows easily that $\square \times \Delta_{L} \times \Delta_{R}$ contains the identical representation just once (octonion multiplication).

As the reader shall recall, the extra polytopes in $\operatorname{dim} 4$ owe their existence to the fact that the $\operatorname{Spin}(4)$ is a direct product, hence the Rot groups are also a direct product of spaces: none of this happens in $\operatorname{Spin}(8)$, so NO new regular polytopes arise. Nevertheless, there are important quasi-regular lattices in $\mathbb{R}^{8}$ !. See e.g. [17.

However, the octonions do arise! How? Precisely because of the triality in the the sense of Adams! As octonions are not our major object of study, we just sum up some important properties: we repeat the conventional construction (Hamilton, Cayley, Graves): namely, take three independent anti-involutive units $e_{1}, e_{2}$ and $e_{3}$, so $e_{1}{ }^{2}=-1$ etc; and force all products to be still anti-involutive, namely $e_{4}:=e_{1} e_{2}, e_{5}=e_{2} e_{3}$ and $e_{6}=e_{3} e_{1}$ : so they have to ANTIcommute. But there is an extra one, $e_{7}:=e_{1}\left(e_{2} e_{3}\right)$ : for this to be also antiinvolutive, associativity is lost: $e_{1}\left(e_{2} e_{3}\right)=-\left(e_{1} e_{2}\right) e_{3}$ ! And we have a base for the imaginary octonions: $e_{1}, \ldots, e_{7}$. With unit $1=e_{0}$ and all real combinations, we form an algebra which is 8-dim over the reals $\mathbb{R}$; a generic element would be $o=\sum_{i=0}^{7} x_{i} e_{i}$; define the conjugate $\bar{o}$ as in $\mathbb{C}$ or $\mathbb{H}$ : changing the signs but of first term. Then for $o \neq 0$ the norm $\bar{o} o=\mathcal{N}(o)$ is real $\neq 0$, so define the inverse as $o^{-1}=o / \mathcal{N}(o)$, and one has the (nonassociative, noncommutative) division algebra of the octonions!

In particular, norm-one octonions form a multiplicative structure (with inverse) in $S^{7}$, but not a group because of the lack of associativity. And it is satisfactory that, back in 1958, Bott and Milnor proved [15, by topological arguments, that $S^{0}\left(=Z_{2}=O(1)\right), S^{1}(\approx \mathrm{U}(1)=\mathrm{SO}(2)), S^{3}(\approx S q(1)=S U(2)=\operatorname{Spin}(3))$ and $S^{7}$ were the only parallelizable spheres, clearly associated, respectively, to $\mathbb{R}, \mathbb{C}, \mathbb{H}$ and (O). 


\section{Final comments}

There are several related things we left behind. For example, tessellations in other spaces: spheres $S^{n}$ are characterized by being of constant positive curvature and hence they are compact. What about other constant curvature spaces, in concrete flat (i.e. $\mathbb{R}^{n}$ ) and negative curvature (e.g. the hyperbolic space $H^{n}$ )? In the mathematical literature they are called "maximally symmetric spaces" [16]. We just recall a couple of well-known results:

- a) In $\mathbb{R}^{2}$, we already realized the regular tessellation condition $\frac{1}{p}+\frac{1}{q}=\frac{1}{2}$, with the three solutions by triangles, squares and hexagons: it is easy to connect the three with the special character of the rotation isometry group, $\mathrm{E}^{+}(2)=\mathbb{R}^{2} \rtimes \mathrm{SO}(2)$, which is nilpotent (semidirect product of two abelian groups): In any other dimension $\neq 4$, only (hyper-)cubes $H_{n}$ tessellate the Euclidean space (the 24 -cell and the dual $H_{4}^{*}$ tessellate $\mathbb{R}^{4}$ also).

- b) For $H^{2}$, hyperbolic, the restriction condition is $\frac{1}{p}+\frac{1}{q}<\frac{1}{2}$, with infinite solutions, like e.g. $\{7,3\}$ : heptagons, three per vertex; as the space is non-compact, with arbitrary (constant negative) curvature, there are infinite tessellations.

Are there other manifolds which can support regular polytopes? For example, what about symmetric rank-one spaces, like $C P^{n}$ ? At the moment, we have nothing (new) to say about this situation, but please check [7.

As a final reference, the review by Conway and Sloane [17] contains much material related to the one dealt with here.

\section{References}

[1] Coxeter, H.S.M.: Regular Polytopes. Dover N.Y. 1973.

[2] Stillwell, J.: Exceptional objects in Mathematics. Am. Math. Month. 105, 850, (1998).

[3] Coxeter, H.S.M.: The Beauty of Geometry (12 Essays). Dover, N.Y. 1991.

[4] Coxeter, H.S.M.: Regular Complex Polytopes. Cambridge U.P. 1974.

[5] Björner, A. and Brenti, F.: Combinatorics of Coxeter groups. Springer, Berlin 2005.

[6] Conway, J.H, and Smith, D.A.: On Quaternions and Octonions. A. K. Peters, Oxford 2003.

[7] McMullen, P. and Schulte, E.: Abstract Regular Polytopes. Cambridge U.P. 2002.

[8] Conway et al.: Atlas of Finite Groups. Oxford U.P. 1985.

[9] Tondeur, P.: Introduction to Lie groups and transformation groups, Lecture Notes in Math. 7, Springer, Berlin 1967, Appendix.

[10] Jacobson, N.: Lie algebras. J. Wiley, N.Y. 1967. 
[11] Steenrod, N.: Topology of Fibre Bundles. Princeton U.P. 1951.

[12] Cartan, É.: La Theórie des Spineurs (2 Vols.). Hermann, Paris 1938.

[13] Boya, L.J.: The geometry of Lie Groups. Rep. Math. Phys. 30, 149, (1991).

[14] Adams, J.F.: Lectures on Exceptional Lie Groups, U. Chicago Press 1996.

[15] Milnor, J. and Stasheff, S.: Characteristic classes. Princeton U.P. 1972.

[16] Helgason, S.: Differential Geometry and Symmetric Spaces. Academic Press, Waltham 1976.

[17] Conway, J.H. and Sloane, N. J. A.: Sphere Packing, Lattices and Groups. Springer, Berlin 1988.

[18] Coxeter, H.S.M: Introduction to Geometry. J.Wiley, N.Y. 1989.

[19] Boya, L.J. Rivera, C. Grupos Abelianos finitos. La Gaceta Matemática Española 13, 229, (2010).

[20] See, e.g.

Milnor, J.: Morse theory. Princeton U.P. 1969. 